\title{
Soil-Site Suitability and Production Potential Evaluation of Chickpea (Cicer arietinum) under Arid Climate of Western Rajasthan, India
}

\author{
R.L. Meena ${ }^{1 *}$, T.P. Verma, R.S. Singh, P.C. Moharana, Sunil Kumar, \\ Mahaveer Nogiya, B.L. Tailor, R. Singh and S.K. Singh ${ }^{2}$ \\ ${ }^{1}$ ICAR-National Bureau of Soil Survey and Land Use Planning, Regional Centre, \\ Udaipur- 313001 (RJ), India \\ ${ }^{2}$ ICAR-National Bureau of Soil Survey and Land Use Planning, \\ Nagpur- 440033 (MH), India \\ *Corresponding author
}

\section{Keywords \\ Soil site suitability, Evaluation, Chickpea and Limitations \\ Article Info \\ Accepted: \\ 20 March 2019 \\ Available Online: \\ 10 April 2019}

\section{A B S T R A C T}

A detailed soil survey was undertaken in central state farm Jetsar, Sri Ganganagar, Rajasthan, India representing the arid climate with the aim of to assess the status and potential of land resources for the suitability and production potential of soils for chickpea. The area of the farm has been divided into three major landforms viz., sand dunes, reclaimed sand dunes and aeofluvial flood plain. Based on the variation in physiography and landforms, eight soil pedons were identified in the farm. Pedon P1 \& P2 occurring on sand dune and reclaimed sand dune which are sandy deep, mixed, calcareous, Typic Torripsmments whereas pedons from P3 to P8 occurring on aeofluvial flood plains which are deep, calcareous, coarse loamy to fine silty Typic/Sodic/Fluventic Haplocambids and Oxyaquic Torrifluvents. Soils of the farm belong to very deep category ranged from 135$195 \mathrm{~cm}$, sandy to clay textured developed on aeolian and alluvium parent material. These soils are moderately alkaline $(8.25 \mathrm{pH})$ to strongly alkaline $(9.56 \mathrm{pH})$, very low $(0.02 \%)$ to low $(0.29 \%)$ in organic carbon, non saline (EC 0.13$)$ to strongly saline (EC $7.50 \mathrm{dsm}-1$ ), low $(1.95 \%)$ to high $(19.51 \%)$ in calcium carbonate. Further, soils were low in available nitrogen, low to medium in available phosphorus, low to medium in available potassium whereas soils were low in available $\mathrm{Fe}$ and $\mathrm{Mn}$, and high in available $\mathrm{Zn}$ and $\mathrm{Cu}$. Soil has been assessed for suitability of chickpea as per the criteria given by Naidu et al 2004 . Soils of the pedon P3, P6 and P7 were moderately suitable, whereas pedon P5 was marginally suitable for the cultivation of chickpea. Soils of pedon P1, P2, P4 and and P8 were not suitable for the chickpea cultivation due to extreme values of texture, $\mathrm{pH}$ and organic carbon respectively. Potentially soils of pedon P3, P5, P6 and P7 were moderately suitable in contrast to P2 and P8 which are marginally suitable. Yield of the farm can increased $9-36 \%$ with soil and fertility related managements.

\section{Introduction}

Chickpea is an important leguminous crop because it has commercial, trade and dietary value in India, which contain around $>20 \%$ protein and rich in essential amino acids such as lysine, isoleucine, arginine, and total aromatic amino acids (Naser Maheri-Sis et al, 
2008)). It is a major rainfed rabi pulse crop of arid part of Rajasthan which accounts around $19 \%$ area and contribute $17 \%$ production of the country with productivity of $8.5 \mathrm{q} / \mathrm{ha}$ (Agricultural statistics, 2014). Increasing population of the country enforced the intensive use of natural resource to feed its inhabitants. Assorted use of land resources due to population pressure, expand their degradation and in-turn decline in soil fertility, degradation and finally poor factorproductivity.

This adventitious use of the natural resources is due to lack of information about the soilsite suitability of crops as conditional by climate, topography and management level (Sehgal, 1991). However, growing the crops without proper consideration of soil and site characteristics has observed with continuously low yield with soil health downturn. We need to be using the natural resources according to their capacity to satisfy the needs of its inhabitants. This can be achieved through proper investigation of land resources and their scientific evaluation.

Information on soil-site suitability of chickpea crop in different landforms of central state farm as well as for entire western Rajasthan is scanty. Hence, it is desirable that the chickpea crop should be grown as per suitability in different kind of soils as well as climate and physiography. Several workers have worked out the suitability of soils for various crops such as wheat (Sharma, 1999), cotton (Mandal et al., 2002) and sorghum (Pakhan et al., 2010). Considering this, soil-site requirement of chickpea for the region was developed talking into account the available literature and field and local experience as suggested by Naidu et al., (2006) and FAO (1976). In the present study, an attempt has been made to evaluate "Soil-site suitability for chickpea in central state farm, Jetsar".

\section{Materials and Methods}

\section{Location and climate}

Central state farm (Unit of national seed corporation) is situated in Sri Raisinghnagar and Sri Bijaynagar block of Sri Ganganagar district in Rajasthan, which falls in western dry zone lies between $24^{\circ} 39^{\prime} 26^{\prime \prime}$ to $24^{\circ} 44^{\prime} 00^{\prime \prime}$ $\mathrm{N}$ latitude and $74^{\circ} 21^{\prime} 33^{\prime \prime}$ to $74^{\circ} 28^{\prime} 04^{\prime \prime} \mathrm{E}$ longitude. The farm is part of vast former flood plain mixed with aeolian sandy deposits and alluvium parent material. It representing western plain-semi arid transitional plains physiography which representing hot arid western plain marusthali (Thar Desert) of agro-eco sub region (2.1). The area has very scanty and erratic rainfall with extremely hot in summer and cold in winter. The average rainfall of the area is $286 \mathrm{~mm}$ an some years it is negligible. Hence, the study area qualifies for hyperthermic temperature regime. The length of growing period (LGP) of the area is 45-60 days. Out of 5394.35 ha area of farm about $53.6 \%$ ha area under cultivation, $26.40 \%$ under sand dunes, and $20.3 \%$ ha is under cultural waste. The major landforms identified in the area are sand dunes $(15.61 \%)$, reclaimed sand dunes $(33.27 \%)$ and aeo fluvial flood plain (29.3\%). The topography of the farms composed of Deny, undulating uplands and aero-fluvial plains. Major part of the cultivable lands is under canal irrigation. The major area of the farm enjoys the cultivation of chickpea, wheat, pearl millet, guar, mustard and pulses.

\section{Soil sampling and analysis}

Detailed soil survey of the farm conducted using cadastral map as a base (1:4000 scale) during May, 2014. The soil correlation exercise yielded 8 soil pedons in the study area. The pedons were studied on defined land forms for morphological characteristics following the procedure given in Soil Survey 
Staff (2006). Horizon-wise soil samples collected from the typifying pedon and analyzed for their soil genesis, physical, physic-chemical, chemical and nutrient status properties following standard procedures. The soils were classified according to soil taxonomy (Soil Survey Staff, 2006) and generated thematic maps of the farm which represented in Figure 2. Soil pH and electrical conductivity was measured in 1:2.5 soil water suspension using glass electrode $\mathrm{pH}$ meter and conductivity bridge (Jackson, 1973). The organic carbon was determined by rapid titration method (Walkley and Black, 1934) and $\mathrm{CaCO}_{3}$ by rapid titration method (Puri, 1930). The available micronutrients in soil samples were extracted with DTPA $(0.005 \mathrm{M}$ DTPA + 0.01 $\mathrm{M} \mathrm{CaCl}_{2}+0.1 \mathrm{M}$ TEA, pH 7.3) as per the method described by Lindsay and Norvell (1978) and the concentration of $\mathrm{Zn}$, $\mathrm{Fe}, \mathrm{Cu}$ and $\mathrm{Mn}$ in the DTPA-extract was determined using atomic absorption spectrophotometer.

\section{Soil-site suitability evaluation}

The landscape and soil site characteristics were used to evaluate soil suitability for chickpea as per the guidelines given by Naidu et al., (2006) Sys et al., (1991) represented in Figure 1. The land suitability has been assessed by comparing the landscape and soil characteristics with crop requirements at different suitability levels: S1: Highly suitable, S2: Moderately suitable, S3: Marginally suitable, N: Not suitable. Thus, the evaluation was done by comparing the land characteristics with suitability levels of the crop requirement tables (Naidu et al., (2006). The degree of limitations suggested the suitability class of each soil for a particular crop. The potential land suitability subclasses were determined after considering the improvement measures to correct the limitations. Production potential calculation of chickpea has been done based actual yield and yield potential in the suitability class against the maximum attainable yield of the crop in particular area. FAO (1976) has been defined the potential yield against their suitability class which is $0.8-1.0 \%$ for highly suitable (S1), $0.4-0.8 \%$ for moderately suitable (S2), $\quad 0.2-0.4 \%$ for marginally suitable (S3) and 0.0-0.20\% not suitable (N).

\section{Results and Discussion}

\section{Soil characteristics (physical, chemical and fertility)}

The data regard to soil characteristics of dissimilar landforms of the pedon P1 to P8 is adjacent in Table 1 and 2.

\section{Sand dunes (Pedon P1)}

The sand content ranged from 87.10 to 89.32 with the mean value of $88.30 \%$ whereas clay content stretch from 9.12 to $10.93 \%$ with the mean value of $9.90 \%$. The soil $\mathrm{pH}$ fluctuate from 8.58 to 9.12 with the mean value of 8.85 showing the strongly alkaline in reaction which might be due to salt deposition in soil layers due to high temperature and very low rainfall. These findings are similar to those of Sharma and Bhaskar (2003). The organic carbon content varied from 0.02 to $0.12 \%$ (mean $0.06 \%$ ) indicating the soils were very low in organic carbon content. The content of $\mathrm{CaCO}_{3}$ stretch between 2.20 and $6.34 \%$ (mean of $4.15 \%$ ). The EC ranged from 0.14 to $0.22 \mathrm{dsm}^{-1}$ (mean of $0.19 \mathrm{dsm}^{-1}$ ).

The CEC varied from 7.34 to $13.32 \mathrm{cmol}\left(\mathrm{p}^{+}\right)$ $\mathrm{kg}^{-1}$ (mean of $9.35 \mathrm{cmol}\left(\mathrm{p}^{+}\right) \mathrm{kg}^{-1}$ ) with loamy sand texture. Similar observations were also observed by Savalia et al., (2000). This pedon observed with low values of N, P, K, Fe and $\mathrm{Mn}$ whereas $\mathrm{Zn}$ and $\mathrm{Cu}$ content were high in the soils. Moisture retention capacity of the pedon was 8.5 and $3.5\left(\mathrm{~m}^{3} \mathrm{~m}^{-3}\right)$ on 0.03 and $1.5 \mathrm{MPa}$ respectively. 


\section{Reclaimed sand dunes (Pedon P2)}

The values of sand content ranged between 84.9 to $90.13 \%$ with the mean of $87.61 \%$ whereas the clay content ranged from 6.77 to $10.76 \%$ with the mean value of $8.89 \%$. The soil $\mathrm{pH}$ varied from 8.52 to 8.93 with the mean of 8.77 indicating the strongly alkaline in nature. The organic carbon content of soils was very low and ranged between 0.02 and $0.10 \%$ (mean of $0.06 \%$ ) with $\mathrm{CaCO}_{3}$ ranged from 2.20 to $10.12 \%$ (mean of $6.58 \%$ ). The EC varied from 0.13 to $0.21 \mathrm{dsm}^{-1}$ (mean of $0.16 \mathrm{dsm}^{-1}$ ) whereas CEC ranged from 4.62 to $12.77 \mathrm{cmol}\left(\mathrm{p}^{+}\right) \mathrm{kg}^{-1}$ (mean of $8.26 \mathrm{cmol}\left(\mathrm{p}^{+}\right)$ $\mathrm{kg}^{-1}$ ) with loamy sand to loamy sand texture. These findings are similar to those of Sharma and Bhaskar (2003). Pedon P2 observed with low values of $\mathrm{N}, \mathrm{P}, \mathrm{Fe}, \mathrm{Mn}$ with medium availability of $\mathrm{K}$ whereas $\mathrm{Zn}$ and $\mathrm{Cu}$ content was high in the soils. Moisture retention capacity of the pedon was 8.1 and $3.1\left(\mathrm{~m}^{3} \mathrm{~m}^{-3}\right)$ on 0.03 and $1.5 \mathrm{MPa}$ respectively.

\section{Aeo fluvial flood plain (Pedon P3-P8)}

The sand content in pedon P3 varied from 51.1 to $84.05 \%$ (mean of $66.39 \%$ ) whereas the clay content ranged from 9.52 to $19.07 \%$ (mean of $15.07 \%$ ). The soil $\mathrm{pH}$ varies 8.47 to 8.67 with the mean value of 8.58 showing the strongly alkaline in reaction which might be due to salt deposition in soil layers and due to high temperature and very less rainfall in the area. These findings are similar to those of Sharma and Bhaskar (2003). The organic carbon content varied from 0.10 to $0.23 \%$ (mean $0.13 \%$ ) indicating the soils were very low in organic carbon content. The content of $\mathrm{CaCO}_{3}$ ranged between 1.95 to $4.02 \%$ (mean of $3.24 \%$ ). The EC ranged from 0.15 to 0.22 $\mathrm{dsm}^{-1}$ (mean of $0.18 \mathrm{dsm}^{-1}$ ). The CEC varied from 5.31 to $10.93 \mathrm{cmol}\left(\mathrm{p}^{+}\right) \mathrm{kg}^{-1}$ (mean of $8.81 \mathrm{cmol}\left(\mathrm{p}^{+}\right) \mathrm{kg}^{-1}$ ) with loamy sand, sandy loam and sandy clay loam texture. Similar observations were also observed by Savalia $e t$ al., (2010). Pedon P3 registered with low values of $\mathrm{N}, \mathrm{P}, \mathrm{Fe}, \mathrm{Mn}$ with medium availability of $\mathrm{K}$ whereas $\mathrm{Zn}$ and $\mathrm{Cu}$ content was high in the soils. Moisture retention capacity of the pedon was 16.8 and 6.2 $\left(\mathrm{m}^{3} \mathrm{~m}^{-3}\right)$ on 0.03 and $1.5 \mathrm{MPa}$ respectively.

In pedon P4 sand content varied from 75.8 to $87.95 \%$ (mean of $80.61 \%$ ) whereas the clay content ranged from 9.69 to $12.67 \%$ (mean of $10.85 \%$ ). The soil $\mathrm{pH}$ varies 8.25 to 8.70 with the mean value of 8.41 showing the moderately to strongly alkaline in reaction which might be due to salt deposition in soil layers due to high temperature and very less rainfall in the area. These findings are similar to those of Sharma and Bhaskar (2003). The organic carbon content varied from 0.10 to $0.25 \%$ (mean $0.14 \%$ ) indicating the soils were very low in organic carbon content. The content of $\mathrm{CaCO}_{3}$ ranged between 2.32 to $3.79 \%$ (mean of $2.81 \%$ ). The EC ranged from 0.20 to $0.38 \mathrm{dsm}^{-1}$ (mean of $0.27 \mathrm{dsm}^{-1}$ ). The CEC varied from 6.3 to $10.29 \mathrm{cmol}\left(\mathrm{p}^{+}\right) \mathrm{kg}$ ${ }^{1}$ (mean of $7.62 \mathrm{cmol}\left(\mathrm{p}^{+}\right) \mathrm{kg}^{-1}$ ) with loamy sand to sandy loam texture. Similar observations were also observed by Savalia $e t$ al., (2010). Pedon P4 detect with low availability of $\mathrm{N}, \mathrm{P}, \mathrm{Fe}, \mathrm{Mn}$ with medium value of $\mathrm{K}$ whereas $\mathrm{Zn}$ and $\mathrm{Cu}$ content was high in the soils. Moisture retention capacity of the pedon was 10.4 and $25.4\left(\mathrm{~m}^{3} \mathrm{~m}^{-3}\right)$ on 0.03 and $1.5 \mathrm{MPa}$, respectively.

In pedon $\mathrm{P} 5$, the sand content ranged from 16.2 to $77.15 \%$ (mean of $53.0 \%$ ) whereas clay content ranged from 11.03 to $39.53 \%$ (mean of $23.24 \%$ ). The soil exhibited strongly alkaline in reaction with mean $\mathrm{pH}$ value of 8.65. The organic carbon was low in this profile with the mean value of $0.11 \%$. The EC and CEC values observed with a mean of 0.28 $\mathrm{dsm}^{-1}$ and $19.53 \mathrm{cmol}\left(\mathrm{p}^{+}\right) \mathrm{kg}^{-1}$ ) respectively. The ESP value was varied from 4.4 to $12.1 \%$ with a mean value of $7.57 \%$. 
Table.1 Physical and chemical characteristic of the soils of CSF, Jetsar, Sri Ganganagar

\begin{tabular}{|c|c|c|c|c|c|c|c|c|c|c|c|}
\hline \multirow[t]{4}{*}{ Horizon } & \multirow{4}{*}{$\begin{array}{c}\text { Depth } \\
\text { (cm) }\end{array}$} & & & \multicolumn{8}{|c|}{ Size, class and particle diameter (mm) } \\
\hline & & \multicolumn{3}{|c|}{ Total (\%) } & \multirow[b]{2}{*}{$\begin{array}{l}\text { O.C. } \\
(\%)\end{array}$} & \multirow[b]{2}{*}{$\underset{(<2 \mathrm{~mm})(\%)}{\mathrm{CaCO}_{3}}$} & \multirow[b]{2}{*}{$\begin{array}{c}\mathbf{p H} \\
(1: 2.5) \mathrm{H}_{2} \mathrm{O}\end{array}$} & \multirow[b]{2}{*}{$\begin{array}{l}\text { E.C. }(1: 2.5) \\
\mathrm{H}_{2} \mathrm{O}\left(\mathrm{dsm}^{-1}\right)\end{array}$} & \multirow[b]{2}{*}{$\begin{array}{l}\text { CEC } \\
\operatorname{Cmol}\left(p^{+}\right) \mathbf{k g}^{-1}\end{array}$} & \multirow[b]{2}{*}{$\begin{array}{l}\text { ESP } \\
(\%)\end{array}$} & \multirow[b]{2}{*}{ Texture } \\
\hline & & Sand & Silt & Clay & & & & & & & \\
\hline & & $(2.0-0.05)$ & $(0.05-0.002)$ & $(<.002)$ & & & & & & & \\
\hline \multicolumn{12}{|c|}{ Pedon $1\left(2^{0} 23^{\prime} 03.1 \mathrm{~N}, 7^{0} 31^{\prime} 3.4 \mathrm{E}\right)$ Mixed (cal), hyperthermic, Typic Torripsamments } \\
\hline A & $0-30$ & 89.32 & 0.95 & 9.73 & 0.06 & 2.20 & 8.58 & 0.14 & 8.97 & - & ls \\
\hline C1 & $30-60$ & 89.05 & 1.83 & 9.12 & 0.04 & 3.17 & 8.66 & 0.20 & 7.88 & - & 1s \\
\hline C2 & $60-90$ & 87.77 & 2.16 & 10.07 & 0.02 & 3.90 & 8.80 & 0.19 & 9.24 & - & 1s \\
\hline C3 & $90-120$ & 87.1 & 1.97 & 10.93 & 0.06 & 5.12 & 9.07 & 0.21 & 13.32 & - & 1s \\
\hline C4 & $120-165$ & 88.26 & 2.08 & 9.66 & 0.12 & 6.34 & 9.12 & 0.22 & 7.34 & - & 1s \\
\hline \multicolumn{12}{|c|}{ Pedon $2\left(29^{0} 24^{\prime} 18.7\right.$ N, $7^{\circ} 30^{\prime} 7.8$ E) Mixed (cal), hyperthermic, Typic Torripsamments } \\
\hline $\mathbf{A}$ & $0-18$ & 89.42 & 3.81 & 6.77 & 0.10 & 2.46 & 8.52 & 0.13 & 4.62 & - & $\mathrm{s}$ \\
\hline C1 & $18-42$ & 90.13 & 2.92 & 6.95 & 0.02 & 2.20 & 8.93 & 0.13 & 5.16 & - & $\mathrm{s}$ \\
\hline C2 & $42-80$ & 84.9 & 4.34 & 10.76 & 0.10 & 10.12 & 8.78 & 0.20 & 11.14 & - & is \\
\hline C3 & $80-130$ & 87.14 & 3.08 & 9.78 & 0.04 & 9.83 & 8.74 & 0.21 & 7.61 & - & 1s \\
\hline C4 & $130-180$ & 86.47 & 3.32 & 10.21 & 0.04 & 8.29 & 8.86 & 0.15 & 12.77 & - & 1s \\
\hline \multicolumn{12}{|c|}{ Pedon $3\left(29^{0} 22^{\prime} 46.9 \mathrm{~N}, 7^{0} 32^{\prime} 42.6 \mathrm{E}\right)$ Coarse loamy, mixed (cal) hyperthermic, Typic Haplocambids } \\
\hline Ap & $0-20$ & 84.05 & 6.43 & 9.52 & 0.23 & 2.68 & 8.47 & 0.18 & 7.29 & - & 1s \\
\hline Bw1 & $20-48$ & 74.49 & 14.82 & 11.49 & 0.10 & 1.95 & 8.56 & 0.17 & 5.31 & - & sl \\
\hline Bw2 & $48-70$ & 70.05 & 16.24 & 13.71 & 0.12 & 3.72 & 8.60 & 0.22 & 10.11 & - & sl \\
\hline Bw3 & $70-105$ & 62.15 & 22.38 & 15.47 & 0.12 & 3.54 & 8.62 & 0.16 & 9.61 & - & sl \\
\hline Bw4 & $105-135$ & 56.5 & 24.43 & 19.07 & 0.12 & 3.54 & 8.55 & 0.15 & 9.61 & - & sl \\
\hline BC & $135-170$ & 51.1 & 27.73 & 21.17 & 0.10 & 4.02 & 8.67 & 0.19 & 10.93 & - & $\mathrm{scl}$ \\
\hline Pedon 4 & $1 ' 58.1 \mathrm{~N}$, & $\left.{ }^{0} 30^{\prime} 44.5 E\right)$ & Sandy over co & rse loamy & $\operatorname{mixed}($ & 1), Oxyaquic 1 & orrifluvents & & & & \\
\hline Ap & $0-23$ & 77.35 & 12.96 & 9.69 & 0.25 & 3.79 & 8.40 & 0.38 & 10.29 & - & sl \\
\hline C1 & $23-45$ & 87.95 & 2.18 & 9.87 & 0.12 & 2.37 & 8.70 & 0.20 & 6.43 & - & 1s \\
\hline C2 & $45-65$ & 84.15 & 6.13 & 9.72 & 0.10 & 2.32 & & & 6.30 & - & 1s \\
\hline C3 & $65-100$ & 82.29 & 7.15 & 10.56 & 0.13 & 2.86 & 8.25 & 0.23 & 7.77 & - & sl \\
\hline C4 & $100-130$ & 76.14 & 11.19 & 12.67 & 0.10 & 2.81 & 8.29 & 0.32 & 7.62 & - & sl \\
\hline C5 & $130-155$ & 75.8 & 11.6 & 12.6 & 0.13 & 2.68 & 8.43 & 0.20 & 7.29 & - & sl \\
\hline Pedon 5 & $22^{\prime} 31.3 \mathrm{~N}$, & 035’30.1 E) & Fine silty, mix & d (cal.), h & perther & ic, Sodic Hapl & cambids & & & & \\
\hline Ap & $0-22$ & 77.15 & 11.82 & 11.03 & 0.19 & 4.76 & 8.58 & 0.47 & 11.92 & 5.8 & sl \\
\hline Bw1 & $22-50$ & 71.25 & 12.08 & 16.67 & 0.12 & 5.00 & 8.70 & 0.28 & 12.59 & 12.1 & sl \\
\hline Bw2 & $50-72$ & 68.96 & 13.47 & 17.57 & 0.10 & 6.22 & 8.60 & 0.24 & 15.9 & 10.4 & sl \\
\hline Bk1 & $72-103$ & 47.2 & 27.61 & 25.19 & 0.08 & 15.73 & 8.66 & 0.22 & 25.75 & 4.7 & $\mathrm{scl}$ \\
\hline Bk2 & $103-135$ & 55.87 & 22.23 & 21.9 & 0.08 & 12.81 & 8.72 & 0.24 & 28.8 & 4.4 & $\mathrm{scl}$ \\
\hline Bk3 & $135-175$ & 16.2 & 44.27 & 39.53 & 0.10 & 19.51 & 8.68 & 0.28 & 29.02 & 5.3 & sicl \\
\hline IIC & $175-195$ & 34.43 & 34.75 & 30.82 & 0.08 & 15.73 & 8.64 & 0.26 & 12.75 & 10.3 & $\mathrm{cl}$ \\
\hline Pedon 6 & $22^{\prime} 37.7 \mathrm{~N}$, & ${ }^{0} 30^{\prime} 46.0$ E) & Fine loamy ov & r coarse 1 & amy, $m$ & ed (cal), hyper & hermic, Fluve & tic Haplocaml & & & \\
\hline Ap & $0-15$ & 59.69 & 20.31 & 20.00 & 0.29 & 9.15 & 8.64 & 0.28 & 5.16 & 5.4 & sl \\
\hline Bw1 & $15-45$ & 49.54 & 27.16 & 23.30 & 0.10 & 13.66 & 8.76 & 0.17 & 3.53 & 3.7 & scl \\
\hline Bw2 & $45-75$ & 15.41 & 61.17 & 23.42 & 0.10 & 9.51 & 8.78 & 0.18 & 9.78 & 1.3 & sil \\
\hline Bw3 & $75-105$ & 79.66 & 8.83 & 11.51 & 0.02 & 10.00 & 8.82 & 0.16 & 7.88 & 1.6 & sl \\
\hline Bw4 & $105-133$ & 53.34 & 33.02 & 13.64 & 0.06 & 12.68 & 8.86 & 0.22 & 5.17 & 4.4 & sl \\
\hline C1 & $133-152$ & 88.74 & 1.04 & 10.22 & 0.02 & 7.56 & 8.92 & 0.19 & 4.62 & 4.8 & 1s \\
\hline $\mathrm{C2}$ & $152-170$ & 81.83 & 8.26 & 9.91 & 0.06 & 7.07 & 9.01 & 0.21 & 6.79 & 3.7 & ls \\
\hline Pedon 7 & '3'16.8 N,' & 34’03.4 E) & Coarse loamy & mixed (ca & , hyper & ermic, Fluven & c Haplocam & & & & \\
\hline Ap & $0-15$ & 82.26 & 9.4 & 8.34 & 0.19 & 4.02 & 8.69 & 0.27 & 5.83 & 6.3 & 1s \\
\hline $\mathbf{A E}$ & $15-32$ & 78.8 & 12.06 & 9.14 & 0.23 & 4.02 & 8.54 & 0.20 & 6.56 & 1.8 & sl \\
\hline Bw1 & $32-60$ & 76.34 & 13.36 & 10.3 & 0.10 & 5.49 & 8.73 & 0.17 & 6.16 & 1.8 & sl \\
\hline Bw1 & $60-95$ & 66.22 & 18.74 & 15.04 & 0.08 & 11.59 & 8.60 & 0.22 & 9.16 & 2.1 & sl \\
\hline Bw3 & $95-150$ & 35.44 & 51.03 & 13.53 & 0.06 & 15.49 & 9.06 & 0.32 & 8.15 & 7.7 & sil \\
\hline Bw4 & $150-195$ & 24.56 & 59.3 & 16.14 & 0.12 & 15.49 & 8.75 & 0.59 & 8.34 & 6.1 & sil \\
\hline Pedon 8 (2 & '’5.8 N, 7 & '09.2 E) F & ine silty over & andy, mix & (cal), & perthermic, & dic Haploc & & & & \\
\hline $\mathbf{A}$ & $0-23$ & 80.82 & 10.16 & 9.02 & 0.13 & 4.37 & 8.73 & 0.23 & 6.87 & 9.8 & ls \\
\hline Bk1 & $23-48$ & 17.69 & 40.08 & 42.23 & 0.10 & 15.78 & 9.27 & 7.50 & 30.87 & 15.3 & sic \\
\hline Bk2 & $48-82$ & 26.36 & 40.58 & 33.06 & 0.12 & 15.53 & 9.33 & 6.70 & 23.21 & 15.0 & $\mathrm{cl}$ \\
\hline II C1 & $82-120$ & 85.29 & 5.57 & 9.14 & 0.10 & 7.53 & 9.56 & 1.09 & 6.45 & 67.4 & ls \\
\hline II C2 & $120-165$ & 79.06 & 12.55 & 8.39 & 0.08 & 8.84 & 9.50 & 1.00 & 6.01 & 68.7 & 1s \\
\hline
\end{tabular}


Table.2 Fertility (weighted mean) and moisture retention status of soils of CSF, Jetsar, Sri Ganga nagar

\begin{tabular}{|c|c|c|c|c|c|c|c|c|c|}
\hline \multirow[t]{3}{*}{ Horizon } & \multicolumn{7}{|c|}{ Available Nutrients (Kg/ha) } & \multicolumn{2}{|c|}{$\begin{array}{c}\text { Moisture Retention } \\
\left(\mathrm{m}^{3} \mathrm{~m}^{-3}\right)\end{array}$} \\
\hline & \multicolumn{3}{|c|}{$\begin{array}{l}\text { Major Nutrients } \\
\left(\mathrm{kg} \mathrm{ha}^{-1)}\right.\end{array}$} & \multicolumn{4}{|c|}{$\begin{array}{l}\text { Micronutrients } \\
\left(\mathrm{mg} \mathrm{kg}^{-1}\right)\end{array}$} & \multirow[t]{2}{*}{$\begin{array}{r}\mathbf{0 . 0 3} \\
\text { MPa }\end{array}$} & \multirow[t]{2}{*}{$\begin{array}{c}1.5 \\
\text { MPa }\end{array}$} \\
\hline & $\mathbf{N}$ & $\mathbf{P}$ & $\mathbf{K}$ & $\mathbf{F e}$ & Mn & $\mathbf{Z n}$ & $\mathrm{Cu}$ & & \\
\hline Pedon 1 & 77.0 & 5.3 & 81.5 & 0.24 & 0.21 & 2.82 & 3.08 & 7.90 & 3.32 \\
\hline Pedon 2 & 67.9 & 7.1 & 112.5 & 0.26 & 0.21 & 2.59 & 2.82 & 8.72 & 3.37 \\
\hline Pedon 3 & 83.4 & 4.7 & 216.0 & 1.02 & 1.33 & 5.72 & 2.55 & 17.11 & 6.32 \\
\hline Pedon 4 & 87.5 & 8.9 & 176.7 & 1.23 & 0.86 & 3.06 & 3.81 & 10.60 & 4.30 \\
\hline Pedon 5 & 89.2 & 7.1 & 156.1 & 0.62 & 0.3 & 5.84 & 2.89 & 20.92 & 10.95 \\
\hline Pedon 6 & 82.2 & 5.1 & 145.9 & 0.54 & 0.31 & 2.68 & 2.82 & 15.86 & 6.82 \\
\hline Pedon 7 & 79.5 & 6.7 & 129.0 & 0.44 & 0.37 & 3.83 & 2.37 & 19.08 & 5.71 \\
\hline Pedon 8 & 74.4 & 11.4 & 146.6 & 0.35 & 0.43 & 3.85 & 4.69 & 18.62 & 8.96 \\
\hline
\end{tabular}

Table.3 Soil-site suitability criteria (crop requirements) for chickpea

\begin{tabular}{|c|c|c|c|c|c|c|}
\hline \multicolumn{3}{|c|}{ Soil-site characteristics } & \multicolumn{4}{|l|}{ Rating } \\
\hline & & Unit & $\begin{array}{l}\text { Highly } \\
\text { suitable S1 }\end{array}$ & $\begin{array}{l}\text { Moderately suitable } \\
\text { S2 }\end{array}$ & $\begin{array}{l}\text { Marginally suitable } \\
\text { S3 }\end{array}$ & $\begin{array}{l}\text { Not suitable } \\
\mathrm{N}\end{array}$ \\
\hline \multirow[t]{2}{*}{$\begin{array}{l}\text { Climatic } \\
\text { regime }\end{array}$} & $\begin{array}{l}\text { Mean temperature in } \\
\text { growing season }\end{array}$ & ${ }^{\circ} \mathrm{C}$ & $20-25$ & $15-19$ & $\begin{array}{l}5-45 \\
26-30\end{array}$ & $\begin{array}{l}>30 \\
<5\end{array}$ \\
\hline & Total rainfall & $\mathrm{mm}$ & $800-1000$ & $600-800$ & $400-600$ & $<400$ \\
\hline $\begin{array}{l}\text { Land } \\
\text { quality }\end{array}$ & \multicolumn{6}{|l|}{ Land characteristics } \\
\hline \multirow[t]{2}{*}{$\begin{array}{l}\text { Moisture } \\
\text { availability }\end{array}$} & $\begin{array}{l}\text { LGP for short duration } \\
\text { verities }\end{array}$ & Days & $>100$ & $90-100$ & $70-90$ & $<70$ \\
\hline & $\begin{array}{l}\text { LGP for long duration } \\
\text { verities }\end{array}$ & Days & $>150$ & $120-150$ & $90-120$ & $<90$ \\
\hline $\begin{array}{l}\mathrm{O}_{2} \\
\text { availability } \\
\text { in roots }\end{array}$ & Soil drainage & class & Well drained & $\begin{array}{l}\text { Moderately well drained, } \\
\text { imperfectly drained }\end{array}$ & $\begin{array}{l}\text { Poorly drained, } \\
\text { excessively drained }\end{array}$ & $\begin{array}{l}\text { Very poorly } \\
\text { drained }\end{array}$ \\
\hline \multirow{2}{*}{$\begin{array}{l}\text { Nutrient } \\
\text { availability }\end{array}$} & Texture & class & 1, sil, cl, scl & sic, sicl, c & $\mathrm{sl}, \mathrm{c}>60 \%$ & - \\
\hline & $\mathrm{pH}$ & $1: 2.5$ & $6.0-7.5$ & $7.6-8.0,5.5-5.7$ & $8.1-9.0,4.5-5.4$ & $>9.0$ \\
\hline \multirow{2}{*}{$\begin{array}{l}\text { Rooting } \\
\text { conditions }\end{array}$} & Effective soil depth & $\mathrm{cm}$ & $>75$ & $51-75$ & $25-50$ & $<25$ \\
\hline & Coarse fragments & $\begin{array}{l}\text { Vol } \\
\%\end{array}$ & $<15$ & $15-35$ & $>35$ & - \\
\hline \multirow[t]{2}{*}{$\begin{array}{l}\text { Soil } \\
\text { toxicity }\end{array}$} & $\begin{array}{l}\text { Salinity (EC saturation } \\
\text { extract) }\end{array}$ & $\mathrm{dS} / \mathrm{m}$ & $<1.0$ & $1.0-2.0$ & $>2.0$ & - \\
\hline & Sodicity (ESP) & $\%$ & $<10$ & $10-15$ & $>15$ & - \\
\hline $\begin{array}{l}\text { Erosion } \\
\text { hazard }\end{array}$ & Slope & $\%$ & $<3$ & $3-5$ & $5-10$ & - \\
\hline
\end{tabular}

Source: Naidu et al., 2006 
Table.4 Suitability analyses of chickpea for CSF farm, Jetsar, Sri Ganga nagar

\begin{tabular}{|c|c|c|c|c|c|c|c|c|c|c|c|c|c|c|}
\hline Pedon & Climate & Slope & Drainage & Texture & Dep. & $\mathrm{CaCO}_{3}$ & pH & EC & O.C. & $\begin{array}{l}\text { Actual land } \\
\text { suitability } \\
\text { class }\end{array}$ & $\begin{array}{l}\text { Potential } \\
\text { land } \\
\text { suitability } \\
\text { class }\end{array}$ & $\begin{array}{l}\text { Actual } \\
\text { Yield } \\
\text { (q/ha) }\end{array}$ & $\begin{array}{c}\text { Potential } \\
\text { Yield } \\
\text { (q/ha) }\end{array}$ & $\begin{array}{c}\text { Yield gap } \\
(\%)\end{array}$ \\
\hline Pedon 1 & S3 & S3 & S3 & $\mathrm{N}$ & $\mathrm{S} 1$ & $\mathrm{~S} 1$ & S3 & $\mathrm{S} 1$ & $\mathrm{~N}$ & $\mathrm{~N}$ & $\mathrm{~N}$ & $2(9)$ & $4(18)$ & $9 \%$ \\
\hline Pedon 2 & S3 & $\mathrm{S} 1$ & S3 & S3 & $\mathrm{S} 1$ & $\mathrm{~S} 1$ & $\mathrm{~N}$ & $\mathrm{~S} 1$ & $\mathrm{~N}$ & $\mathrm{~N}$ & S3sw & $5(23)$ & $8(36)$ & $13 \%$ \\
\hline Pedon 3 & S3 & $\mathrm{S} 1$ & $\mathrm{~S} 1$ & S3 & $\mathrm{S} 1$ & $\mathrm{~S} 1$ & S3 & $\mathrm{S} 1$ & $\mathrm{~N}$ & S2fs & $\mathrm{S} 2 \mathrm{~s}$ & $14(64)$ & $17(77)$ & $13 \%$ \\
\hline Pedon 4 & S3 & $\mathrm{S} 1$ & $\mathrm{~S} 2$ & S3 & $\mathrm{S} 1$ & $\mathrm{~S} 1$ & $\mathrm{~N}$ & $\mathrm{~S} 1$ & $\mathrm{~N}$ & $\mathrm{~N}$ & $\mathrm{~N}$ & $2(9)$ & $4(18)$ & $9 \%$ \\
\hline Pedon 5 & S3 & $\mathrm{S} 1$ & $\mathrm{~S} 2$ & $\mathrm{~S} 2$ & $\mathrm{~S} 1$ & $\mathrm{~S} 1$ & S3 & $\mathrm{S} 1$ & $\mathrm{~N}$ & S3fs & S2fs & $9(41)$ & $17(77)$ & $36 \%$ \\
\hline Pedon 6 & S3 & $\mathrm{S} 1$ & $\mathrm{~S} 1$ & S3 & $\mathrm{S} 1$ & $\mathrm{~S} 1$ & S3 & $\mathrm{S} 1$ & $\mathrm{~N}$ & S2fs & S2s & $12(55)$ & $17(77)$ & $22 \%$ \\
\hline Pedon 7 & S3 & $\mathrm{S} 1$ & $\mathrm{~S} 1$ & S3 & $\mathrm{S} 1$ & $\mathrm{~S} 1$ & S3 & $\mathrm{S} 1$ & $\mathrm{~N}$ & S2fs & $\mathrm{S} 2 \mathrm{~s}$ & $11(50)$ & $17(77)$ & $27 \%$ \\
\hline Pedon 8 & S3 & $\mathrm{S} 1$ & $\mathrm{~S} 1$ & $\mathrm{~S} 2$ & $\mathrm{~S} 1$ & $\mathrm{~S} 1$ & $\mathrm{~N}$ & $\mathrm{~S} 2$ & $\mathrm{~N}$ & $\mathrm{~N}$ & S3fs & $4(18)$ & $8(36)$ & $18 \%$ \\
\hline
\end{tabular}

Chickpea maximum attainable yield in the area $=22 \mathrm{q} / \mathrm{h},() \%$ yield of maximum attainable yield, Suitability subclass: $\mathrm{f}$ - soil fertility limitations; s- physical soil limitations; $\mathrm{w}$ - wetness limitations

Fig.1 Location map of central state farm, Jetsar, Sri Ganga nagar

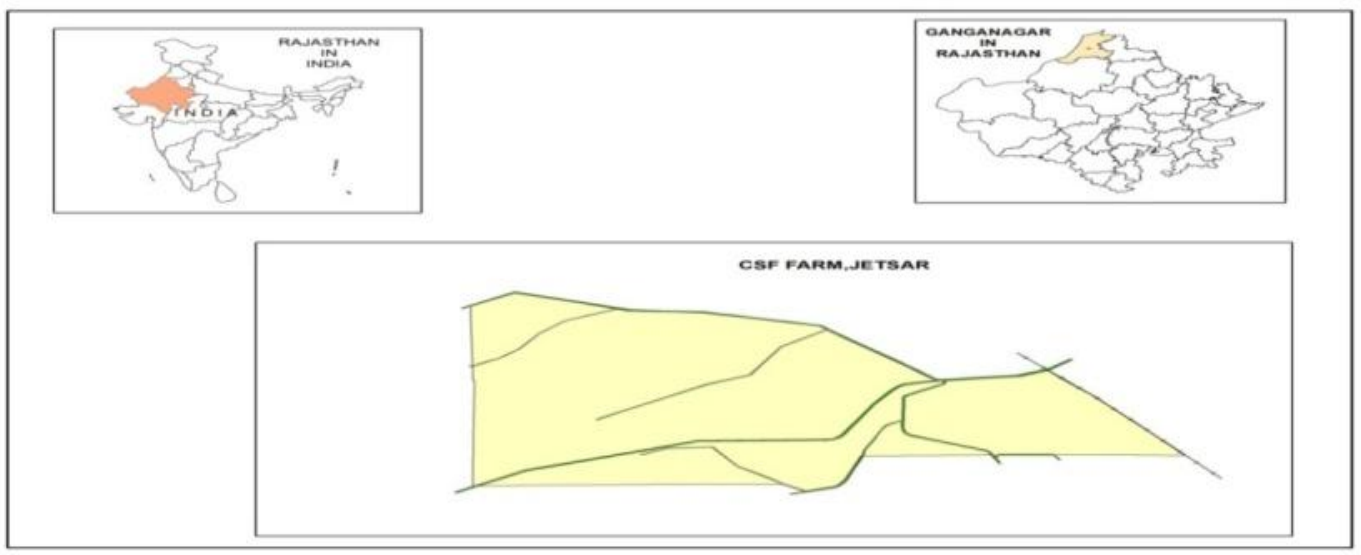


Fig.2 Soil characteristic maps of central state farm, Jetsar, Sri Ganga nagar
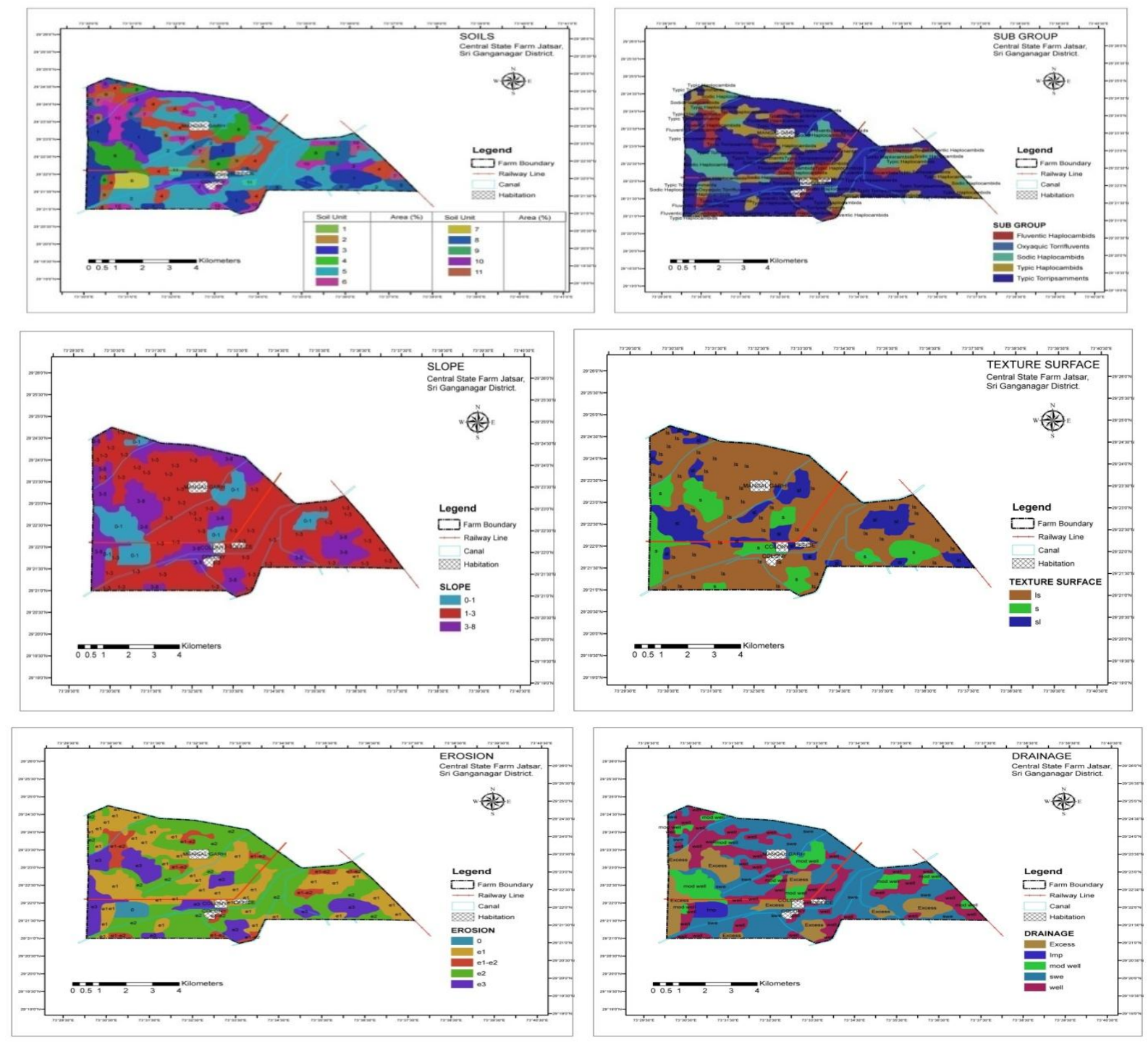

Fig.3 Soil-site suitability map of chickpea for central state farm, Jetsar

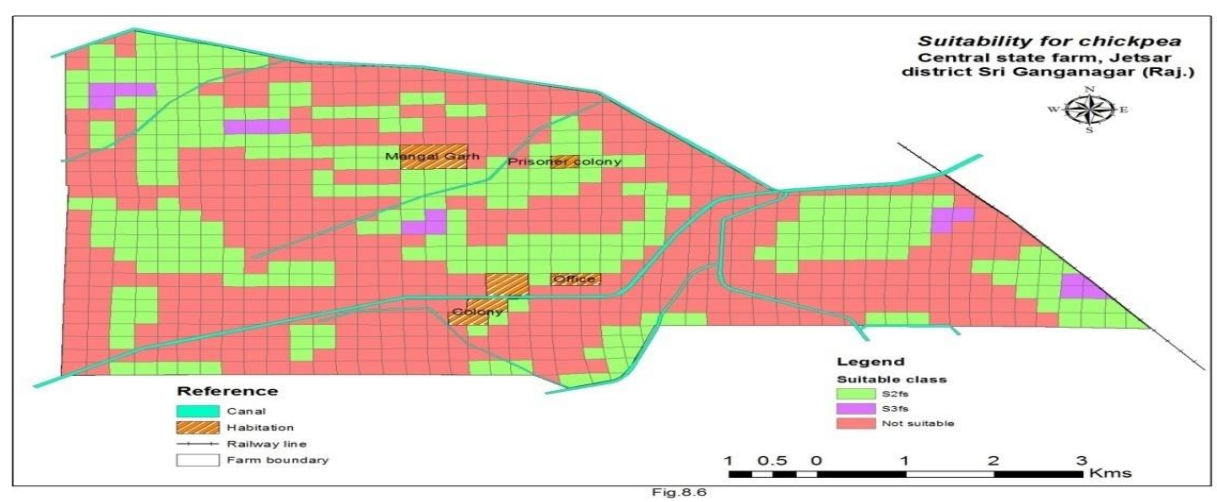

In pedon $\mathrm{P} 7$, the sand content ranged from 24.56 to $82.26 \%$ (mean of $60.60 \%$ ) whereas

clay content ranged from 8.34 to $16.14 \%$ (mean of $12.08 \%$ ). The soil exhibited strongly 
alkaline in reaction with mean $\mathrm{pH}$ value of 8.73. The organic carbon was low in this profile with the mean value of $0.13 \%$. The EC and CEC values observed with a mean of 0.30 $\mathrm{dsm}^{-1}$ and $\left.7.37 \mathrm{cmol}\left(\mathrm{p}^{+}\right) \mathrm{kg}^{-1}\right)$ respectively.

The ESP value was varied from 1.8 to $7.7 \%$ with a mean value of $4.30 \%$. The texture of the profile belongs to sandy loam to silty loam category. Pedon P7 observed with low availability of $\mathrm{N}, \mathrm{Fe}, \mathrm{Mn}$ with medium value of $\mathrm{P}$ and $\mathrm{K}$ whereas $\mathrm{Zn}$ and $\mathrm{Cu}$ content was high in the soils. Moisture retention capacity of the pedon was 16.7 and $5.3\left(\mathrm{~m}^{3} \mathrm{~m}^{-3}\right)$ on 0.03 and $1.5 \mathrm{MPa}$ respectively.

In pedon $\mathrm{P} 8$, the sand content ranged from 17.69 to $85.29 \%$ (mean of $57.84 \%$ ) whereas clay content ranged from 8.39 to $42.23 \%$ (mean of $20.37 \%$ ). The soil exhibited very strongly alkaline in reaction with mean $\mathrm{pH}$ value of 9.28. The organic carbon was low in this profile with the mean value of $0.11 \%$. The EC and CEC values observed with a mean of $3.30 \mathrm{dsm}^{-1}$ and $\left.14.68 \mathrm{cmol}\left(\mathrm{p}^{+}\right) \mathrm{kg}^{-1}\right)$ respectively.

The ESP value was varied from 9.8 to $68.7 \%$ with a mean value of $35.24 \%$. The texture of the profile belongs to loamy sand to silty clay to clay loam. Pedon P8 observed with low availability of $\mathrm{N}, \mathrm{Fe}, \mathrm{Mn}$ with medium value of $\mathrm{P}$ and $\mathrm{K}$ whereas $\mathrm{Zn}$ and $\mathrm{Cu}$ content was high in the soils.

Moisture retention capacity of the pedon was 19.5 and $10.0\left(\mathrm{~m}^{3} \mathrm{~m}^{-3}\right)$ on 0.03 and $1.5 \mathrm{MPa}$ respectively. These findings are completely in agreement to those of Selvaraj and Naidu (2013), Gandhi and Savalia (2014) and Meena et al., (2012).

\section{Soil-site suitability and potential of} chickpea

The soil characteristics of studied pedons used in assessing suitability are presented in table 1 and 2 and suitability map is represented in Figure 3.

\section{Sand dunes (Pedon P1)}

The soils associated with this pedon belong to Typic Torripsamments and currently not suitable $(\mathrm{N})$ for the chickpea cultivation because of major limitations like topography, less organic carbon content and poor soil fertility and soil texture.

\section{Reclaimed sand dunes (Pedon P2)}

Soils of this pedon belong to Typic Torripsamments and currently not suitable for the chickpea cultivation due to extreme soil $\mathrm{pH}$, poor soil fertility and very low organic carbon content but potential of these soils are marginally suitable (S3) with some soil related amendments.

\section{Aeofluvial flood plain (Pedon P3-P8)}

Pedon P3, P6 and P7 which are grouped under Typic/Fluventic Haplocambids are moderately suitable (S2) for chickpea cultivation (Table 3). The major limitations of these pedon are very low organic carbon content, poor soil texture and high soil $\mathrm{pH}$ and poor soil fertility which doesn't allow crop for good germination and growth. Physical and chemical condition of this soil can be improved with some soil amendment practices like gypsum application, farm yard manure application and removal of salts through good irrigation water and yield of chickpea can improved. Pedon P5, which is grouped under Sodic Haplocambids is marginally suitable (S3) for chickpea. These soils showed limitations viz., low organic carbon, high $\mathrm{pH}$ and poor drainage. Pedon $\mathrm{P} 4$ and P8 which are grouped under Oxyaquic Torrifluvents and Sodic Haplocambids respectively are not suitable for the chickpea cultivation due to very low organic carbon, high $\mathrm{pH}$ and poor soil fertility. Pedon P8 is 
potentially marginal suitable with fertility and soil related improvements. The soil fertility of these pedon can be improved by mixing with farm yard manure and green manure year after year.

Actual and potential yield of all the pedon presented in Table 4. Difference between these yields are maximum for pedon $\mathrm{P} 4$ which is $36 \%$ whereas minimum for pedon $\mathrm{P} 1$ and $\mathrm{P} 4$ around $9 \%$. This yield gap among all the pedon varies from 9-36\% which can be reduced with gaining potential yield of chickpea in the central state farm according to particular suitability class with soil and fertility related management practices.

The soil-site suitability evaluation study revealed major limitations of the area such as texture, base saturation, $\mathrm{pH}$ and organic matter. Mixing the gypsum and organic matter in the desert soils with conservation agricultural practices is necessary to improve soil health. The fertility properties such as base saturation, $\mathrm{pH}$ and organic carbon can be improved by addition of organic matter through farm yard manure/compost/green manuring. The soils of pedon P3, P6 and P7 are more suitable to grow the chickpea compared to pedon P1, P2, P4 and P8 soils. Yield gap of $9-36 \%$ has been reduced with soil and fertility related managements in the area. Hence, judicious use of organic manures in combination with inorganic fertilizers in these soils not only pave the way to achieve sustainable yield of chickpea but also to sustain the soil fertility without deterioration for future generations.

\section{References}

Agricultural statistics at a glance (2014). Department of Agriculture \& Cooperation Directorate of Economics \& Statistics, Ministry of Agriculture, Government of India. Page 96.
FAO. (1976). A frame work for land evaluation. Soils Bull 32. FAO, Rome.

Gandhi, G. and Savalia, S.G. (2014). Soil-site suitability evaluation for mustard in calcareous soils of Girnar toposequence in Southern Saurashtra region of Gujarat. Journal of Oilseed Brassica, 5 (2): 128-133.

Jackson, M.L. 1973. Soil Chemical Analysis. Prentice Hall of India Private Limited, New Delhi.

Lindsay, W. L., and W. A. Norvell. (1978). Development of a DTPA Soil Test for Zinc, Iron, Manganese, and Copper. Soil Science Society of America Journal. 42, 421-428.

Mandal, D.K, Kandare, N.C., Mandal, V., and Challa, O. (2002). Assessment of quantitative land evaluation methods and suitability mapping for cotton growing soils of Nagpur district. J Indian Soc Soil Sci, 50, 74-80.

Meena, R.H., Giri, J.D., and Sharma, S.K., (2012). Soil-site Suitability Evaluation for Chickpea in Malwa Plateau of Banswara District, Rajasthan. International Journal of Scientific and Research Publications, Vol. 2(9), 1-6.

Naidu, L.G.K., Ramamurthy, V., Challa, O., Rajendra hedge and Krishnan, P. (2006). Manual soil-site suitability criteria for major crops. NBSS\&LUP Pubbl. 129.

Naser Maheri-Sis, Mohammad Chamani, AliAsghar Sadeghi, Ali MirzaAghazadeh and Abolfaz Aghajanzadeh-Golshani (2008). Nutritional evaluation of kabuli and desi type chickpeas (Cicer arietinum L.) for ruminants using in vitro gas production technique. African Journal of Biotechnology. Vol. 7(16), 29462951.

Pakhan, A.D., Chatterji, S., Sen, T.K., Venugopalan, M.V., Patil, S., and Challa, O. (2010). Use of different 
techniques in evaluation of suitability of shrink swell soils of Nagpur district, Maharashtra for rainfed Sorghum. J Indian Soc Soil Sci, 58, $117-124$.

Puri, A.N. 1930. A new method of estimating total carbonates in soils. Journal of Imperial Agricultural Research, Pusa bulletin 206, 7.

Savalia, S.G., and Gundalia, J.D., (2008). Soil-site suitability evaluation for soybean in Southern Saurashtra region of Gujarat. Legume Research, 31(1), $1-7$.

Savalia, S.G., and Gundalia, J.D., (2009). Soil-site suitability evaluation for Groundnut in Southern Saurashtra region of Gujarat. Legume Research, 32(3), 157-165.

Savalia, S.G., and Gundalia, J.D., (2010). Characterization and evaluation of soil-site suitability for groundnut in the soils of Uben irrigation command area of Saurashtra region in Gujarat. Legume Research, 33(2), 79-86.

Sehgal, J. (1991). Soil-site suitability evaluation for cotton. Agropedology. 1:49-63.

Selvaraj and Naidu, M.V.S. (2013). Land characterization and soil-site suitability for the major crops for
Reniguntamandal in Chittoor district, Andhra Pradesh.

Sharma, J.P. and Bhaskar, B.P. (2003). Variability and Similarity of soils in Rajkot district, Gujarat. Journal of Indian Society of Soil Science. 51(3): 279-287.

Sharma, R.C. (1999). Soil suitability of reclaimed salt affected soils for wheat. Agropedol 9: 59-62.

Soil Survey Staff. (2006). Keys to Soil Taxonomy. $10^{\text {th }}$ edn, USDA, Natural Resources Conservation Service, Washington, DC.

Sys Ir C., E Van Kanst., Debaveye, J and Beernaert, F. (1993). Land evaluation crop requirements. Agricultural publication No. 7, Part III, General, FAO, Rome, Italy.

Sys, C., Van Ranst, E. and debaveye, J. (1991). Land evaluation, Part 2 methods in land Evaluation. Agricultural publications no.7, Belgium.

Walkley, A.J. and Black, C.A. (1934). An estimation of the digestion method for determining soil organic matter and proposed modification of the chromic acid titration method. Soil Science, 37, 29-38.

\section{How to cite this article:}

Meena, R.L., T.P. Verma, R.S. Singh, P.C. Moharana, Sunil Kumar, Mahaveer Nogiya, B.L. Tailor, R. Singh and Singh, S.K. 2019. Soil-Site Suitability and Production Potential Evaluation of Chickpea (Cicer arietinum) under Arid Climate of Western Rajasthan, India. Int.J.Curr.Microbiol.App.Sci. 8(04): 2597-2607. doi: https://doi.org/10.20546/ijcmas.2019.804.302 